\title{
Does Previous Abdominal Surgery Alter the Outcome of Pediatric Patients Subjected to Orthotopic Liver Transplantation?
}

\author{
VALENTIN CUERVAS-MONS, ANTONI RIMOLA, DAVID H. VAN THIEL, JUDITH S. \\ GAVALER, ROBERT R. SCHADE, and THOMAS E. STARZL \\ Clinica Puerta de Hierro, Madrid, Spain; University of Barcelona, Barcelona, Spain; Departments of \\ Medicine and Surgery, University of Pittsburgh, Pittsburgh, Pennsylvania
}

\begin{abstract}
The medical, anesthesia, and surgical records of 89 consecutive pediatric patients who underwent an orthotopic hepatic transplantation procedure at the University of Pittsburgh from February 1981 to May 1984 were reviewed to evaluate the effect of prior abdominal surgery upon the morbidity and mortality of orthotopic liver transplantation in children. Fifty-seven children (group 1) had had prior abdominal surgery, whereas 32 (group 2) had not. The group 1 subjects were younger $(\mathrm{p}<0.001)$, had better prothrombin times $(\mathrm{p}<0.01)$, and better platelet counts $(\mathrm{p}<0.02)$ than did those in group 2. No difference in the duration of anesthesia or intra-operative use of fresh frozen plasma or platelets was evident between the two groups. However, group 1 patients were given more red blood cells intraoperatively than were the group 2 patients $(\mathrm{p}<0.01)$. The group 1 patients had more total postoperative infections ( $p<0.05$ ), which was due solely to a greater number of abdominal infections ( $\mathrm{p}<0.05$ ), but similar total hospital and intensive care unit stays as did the group 2 patients. When those in group 1 were divided into those having a previous Kasai procedure versus those who did not, no differences between the two groups were apparent except for age. Based upon these data, we conclude that prior abdominal surgery does not affect mortality, the duration of hospital or intensive care unit stay, plasma or platelet requirements, and total anesthesia time required for orthotopic liver transplantation, but does enhance the number of red blood cell transfusions and infections, particularly abdominal infections, in children undergoing this procedure.
\end{abstract}

\begin{abstract}
Orthotopic liver transplantation (OLTx) is an enormous surgical procedure that is hampered by the almost inevitable debilitation of potential recipients, the existence of a massive collateral circulation, and the presence of major coagulation abnormalities, all of which are problems present frequently in the patients in whom it is to be applied (1-4). In addition, the presence of adhesions and a variety of anatomic alterations due to previous abdominal operations in potential recipients have been thought to be important complicating factors that make the recipient hepatectomy more difficult than it is in candidates without prior surgical procedures. Transplant surgeons frequently state that technical problems created by prior abdominal surgery, particularly portosystemic shunting procedures and biliary tract surgery, render OLTx a longer and more difficult procedure, with a resultant greater intraoperative blood loss, more frequent postoperative complications, and an overall poorer survival than that which occurs in cases without preexistent surgery.
\end{abstract}

The aim of the present study was to document whether or not prior major abdominal surgery has a deleterious influence upon the outcome and morbidity associated with OLTx when applied to pediatric recipients.

Address requests for reprints to: David H. Van Thiel, M.D., University of Pittsburgh, School of Medicine, 1000J Scaife Hall, Pittsburgh, Pennsylvania 15261. 


\section{Materials and Methods}

The medical, anesthesia, and surgical records of 89 consecutive pediatric patients (18 $\mathrm{yr}$ or younger) who had an OLTx performed at the University of Pittsburgh between February 1981 and May 1984 were evaluated retrospectively to determine the following data: (a) the total anesthesia time and the total amount of intraoperative blood products, used as measures of the difficulty of the surgical procedure; (b) the number of postoperative infections; (c) the frequency of second surgical procedures during the hospital admission when the OLTx procedure was performed; (d) the duration of the hospitalization after the OLTx and the number of days of intensive care required; and (e) the perioperative mortality rate (from time of OLTx to $6 \mathrm{wk}$ after performance of the procedure).

Because not all of these patients underwent retransplantation or other types of reoperation during their hospitalizations, only the anesthesia time and intraoperative blood products used during the initial OLTx procedure were considered. Similarly, in patients undergoing reoperations $(\mathrm{n}=16)$, only infections that occurred during the period between the initial OLTx and the first reoperation were considered. In nonreoperated patients, the frequency of infection was determined for the entire period from the OLTx until discharge.

The patient selection methods, the surgical procedure, the immunosuppressive regime used, and the supportive care offered to these patients have each been described in detail previously (4-7). All of the cases included received comparable clinical management from the same medical and surgical teams, which were constant throughout the study period.

For the purpose of analysis, the 89 patients included in the study were divided into two groups. Group 1 consisted of 57 patients who had had a major abdominal surgical procedure before their OLTx and group 2 consisted of 32 patients who had not. The prior abdominal surgical procedures performed in group 1 patients were portoenterostomy (Kasai procedure, 40 cases), exploratory laparotomy (9 cases), portosystemic shunt ( 3 cases), cholecystectomy (3 cases), splenectomy ( 1 case), and portocholecystostomy ( 1 case). The indications for the OLTx in the patient population studied are listed in Table 1. Actuarial survival curves for each group of patients studied were plotted for a period of $30 \mathrm{mo}$.

The results are expressed as mean \pm standard error of the mean. Student's $t$-test and the $\chi^{2}$ test with the Yates' correction when appropriate were used to analyze the data. A p value $<0.05$ was considered to be significant.

\section{Results}

\section{Characteristics of the Two Patient Populations Studied}

The clinical and laboratory characteristics of the patients in groups 1 and 2 obtained immediately prior to OLTx are shown in Table 2. Both groups were similar in terms of distribution as to sex, incidence of major complications of the primary liver disease, serum bilirubin levels, and albumin concentrations. The patients in groups 1 and 2 differed significantly in terms of age (52.6 \pm 4.0 and $87.8 \pm 10.6$ mo, respectively; $\mathrm{p}<0.001)$, prothrombin time $(14.3 \pm 0.2$ and $16.3 \pm 0.7 \mathrm{~s} ; \mathrm{p}<0.04)$, and platelet count $\left(191 \pm 17 \times 10^{3}\right.$ and $\left.129 \pm 15 \times 10^{3} / \mathrm{mm}^{3} ; \mathrm{p}<0.02\right)$.

\section{Operative Costs of the Procedure in the Patients Studied}

No statistical difference between the two groups was apparent for the duration of anesthesia (698 $\pm 21 \mathrm{~min}$ in group 1 and $643 \pm 23 \mathrm{~min}$ in group 2) and the intraoperative consumption of fresh frozen plasma $(196 \pm 20$ and $135 \pm 23 \mathrm{ml} / \mathrm{kg} \mathrm{wt})$ and platelets $(13 \pm 2$ and $9 \pm 2 \mathrm{ml} / \mathrm{kg}$ 
wt). Patients in group 1 , however, were given a greater amount of packed red blood cells during the procedure $(237 \pm 29 \mathrm{ml} / \mathrm{kg} \mathrm{wt})$ than those in group $2(114 \pm 18 \mathrm{ml} / \mathrm{kg} \mathrm{wt} ; \mathrm{p}<0.01)$.

Three patients from each group were excluded from the analysis of the frequency of postoperative infections as they died intraoperatively ( 1 case in group 1) or within the first 72 $\mathrm{h}$ after the procedure ( 2 cases in group 1 and 3 cases in group 2 ). Twenty-seven $(50 \%)$ of the remaining 54 patients in group 1 developed a postoperative infection, whereas this occurred in only $8(28 \%)$ of the 29 patients in group 2 ( $\mathrm{p}<0.05)$. As shown in Table 3, this difference in frequency of postoperative infections also differed as to the location of the infection. Nineteen patients (35\%) in group 1 developed an abdominal infection, whereas this complication occurred in only 3 patients $(10 \%)$ in group $2(\mathrm{p}<0.05)$. No difference between groups was found with respect to the proportion of patients who developed a postoperative nonabdominal infection (13 in group 1, or 24\%, and 6 in group 2, or $21 \%$ ) or the number of such infections (16 and 8, respectively). The period of time during which the incidence of infections was considered was similar in both groups ( $30 \pm 3$ days in group 1 and $30 \pm 4$ days in group 2).

The perioperative mortality was similar in both groups. Nine patients (16\%) in group 1 and 7 $(21 \%)$ in group 2 died during the period of study. The duration of the hospitalization after OLTx and the number of days for which the intensive care unit was required were similar for the children discharged alive in group 1 ( $54 \pm 4$ and $11 \pm 3$ days, respectively) and those in group 2 (51 \pm 5 and $8 \pm 2$ days) (Figure 1).

To determine whether patients with a pre-OLTx portoenterostomy (Kasai procedure) have a worse perioperative prognosis than do those patients with other types of pre-OLTx surgery, the group 1 patients were divided into two subgroups: group 1A included 40 patients who had been given a prior portoenterostomy, and group 1B included the remaining 17 patients who had not had a portoenterostomy but who had had some other type of abdominal surgery. As shown in Table 4 and Figure 1 no significant differences between groups 1A and 1B were found for pre-OLTx clinical and laboratory data, duration of anesthesia, amount of intraoperative blood products consumed, incidence and types of post-OLTx infection, mortality rate, and duration of hospitalization. The only parameter that was significantly different was age; children of group 1A were younger $(42.6 \pm 3.5 \mathrm{mo})$ than children of group 1 B $(75.4 \pm 19.3$ mo; $\mathrm{p}<0.01)$.

\section{Discussion}

Among the many hepatobiliary disorders for which children are candidates for OLTx (Table $1)$, the most frequent is biliary atresia $(1-4,6,7)$. Unfortunately, both in the precyclosporine era and more recently following the widespread use of cyclosporine, the long-term survival of children receiving liver transplants for biliary atresia has been lower than it has been for recipients with other indications, such as those with liver-based inborn metabolic errors [57). Because liver transplantation in pediatric patients with biliary atresia usually is performed only after prior surgical attempts to restore biliary drainage by portoenterostomy [Kasai procedure) have failed, the lower survival of these patients has been attributed, at least in part, to the difficulties experienced by the surgeon during the recipient hepatectomy, many of which are thought to be caused by the presence of adhesions and other anatomic alterations, which are secondary to the prior performance of the Kasai procedure. It also has been suggested that the creation of a portacaval shunt can render subsequent OLTx more difficult because the residual portal vein obtained after taking clown the shunt, especially side-to-side shunts, may have a suboptimal length and sclerotic walls, which make the subsequent anastomosis with the donor portal vein more difficult $(1,4)$. Similarly, adhesions due to any prior upper abdominal surgery performed before OLTx might also cause difficulties during the recipient hepatectomy 
and might be expected to extract a cost in terms of subsequent infection rates, mortality, and blood product consumption $(1,4)$. Each of these problems has been thought to account for the lower survival rate of children receiving transplants and having had prior abdominal surgical procedures.

Because the many difficulties that a surgical team might have during an OLTx procedure can hardly be analyzed in a retrospective study by nonsurgeons, we instead determined the duration of anesthesia and the volume of blood products consumed intraoperatively in this series of consecutive pediatric patients undergoing OLTx as indirect measures of the same.

The duration of the anesthesia in patients with (group 1) and without (group 2) previous abdominal surgery was amazingly similar. Nonetheless, the volume of red blood cells consumed intraoperatively was twice as much in the group 1 patients as it was in the group 2 patients. These results suggest that, although the total time required to complete the OLTx procedure was not greater in patients who have had previous surgery, there was more intraoperative bleeding in these patients than in those without previous abdominal procedures. Interestingly, this is true despite the fact that the patients in group 1 had better coagulation parameters, as indicated by the lower preoperative prothrombin time and a greater platelet count, than did the patients in group 2. Presumably, because fresh frozen plasma and platelets were transfused only to correct abnormalities detected by repetitive monitoring of the thromboelastogram, similar volumes of fresh frozen plasma and platelets were administered to the two groups studied.

Despite similar operative times, the incidence of postoperative abdominal infections was more than three times greater in the patients who had had previous abdominal surgery (group 1,35\%) than it was in the patients who had not had such prior surgery (group 2, 10\%). Moreover, not only were the measures of severity of the preexistent liver disease quite similar between the two groups (Table 2), so also were the postoperative immunosuppressive regimens (data not given) and the frequency of nonabdominal infections ( $24 \%$ and $21 \%$, respectively). Thus the greater incidence of abdominal infections seen in the group 1 patients cannot be related to a reduced defense against infections, but rather must be ascribed to the fact of having had a previous abdominal operation.

It is of some interest that the patients in group 1 were younger than were the patients in group 2 (Table 2). Because patients in both groups had a similar frequency of nonabdominal infections, the age of the patient at the time of surgery presumably did account for the greater frequency of intraabdominal infections seen in the group 1 subjects. Moreover, as the patients in group 1A, who had had a prior Kasai procedure, were also younger than those in group 1B and the incidence of abdominal and nonabdominal infections was similar in both subgroups, age would not appear to be an important factor determining the increased risk of intraabdominal infection in the group 1 patients. Similarly, it is most unlikely that age played any role in the higher intraoperative consumption of blood cells observed in the group 1 patients. Nonetheless, it should be noted that in patients with advanced liver disease, age and size are not interrelated as they are in the normal population, as children with such disease are disproportionately small due to their illness and its associated problems, such as malabsorption and inanition.

Most interesting is the fact that the patients with pretransplant abdominal surgery (group 1) did not have a greater mortality rate than did the patients without any pretransplant abdominal surgery (group 2). These results indicate that, in spite of the greater intraoperative bleeding and frequency of postoperative abdominal infections, pediatric patients undergoing OLTx after previous abdominal operations have a similar overall outcome as compared with those without such previous abdominal surgery. Thus it can be concluded that the existence of a previous abdominal surgical procedure has to be considered as being only a minor contraindication for 
OLTx - one that increases the morbidity but not the mortality associated with the procedure in pediatric recipients. Additionally, because the intraoperative and postoperative courses of transplanted children with biliary atresia and prior portoenterostomy did not differ from those of children with other hepatobiliary diseases and other types of abdominal surgery, it can be concluded that the existence of a portoenterostomy (Kasai procedure) does not put a child at a greater risk of a postoperative complication, prolonged hospitalization, and death than does the existence of any other types of major abdominal surgery in a child undergoing such surgery. Moreover, because children with biliary atresia with or without a prior Kasai operation are smaller and younger than children with other liver diseases treated with OLTx but have similar survival characteristics, size of the pediatric OLTx recipient, although a technical problem for the operating surgeon, does not appear to adversely affect the morbidity and mortality associated with the procedure.

\section{Acknowledgments}

This work was supported in part by grants from the National Institute of Arthritis, Metabolism, and Digestive Diseases (No. R01 AM32556) and the Gastroenterology Medical Research Foundation of Southwestern Pennsylvania.

\section{Abbreviation used in this paper}

OLTx orthotopic liver transplantation

\section{References}

1. Iwatsuki S, Shaw BW Jr, Starzl TE. Current status of hepatic transplantation. Semin Liv Dis 1983;3:173-80.

2. Scharschmidt BF. Human liver transplantation: analysis of data on 540 patients from four centers. Hepatology 1984;4:95S-101S. [PubMed: 6363266]

3. Starzl TE, Iwatsuki S, Shaw BW Jr, et al. Analysis of liver transplantation. Hepatology 1984;4:47S9S. [PubMed: 6363258]

4. Starzl TE, Iwatsuki S, Van Thiel DH, et al. Evolution of liver transplantation. Hepatology 1982;2:61436. [PubMed: 6749635]

5. Zitelli BJ, Malatack JJ, Gartner JC Jr, et al. Urthotopic liver transplantation in children with hepatic based metabolic disease. Transplant Proc 1983;15:1284-7.

6. Gartner JC Jr, Zitelli BJ, Malatack JJ, et al. Orthotopic liver transplantation in children: two year experience with 47 patients. Pediatrics 1984;74:140-5. [PubMed: 6377219]

7. Zitelli, BJ.; Gartner, JC., Jr; Malatack, JJ., et al. Pediatric liver transplantation with cyclosporine and steroids. Neonatal choleslasis: causes, syndromes, therapies; Report of the 87th Ross Conference on Pediatric Research; Columbus, Ohio: Ross Laboratories; 1984. p. 132-7. 


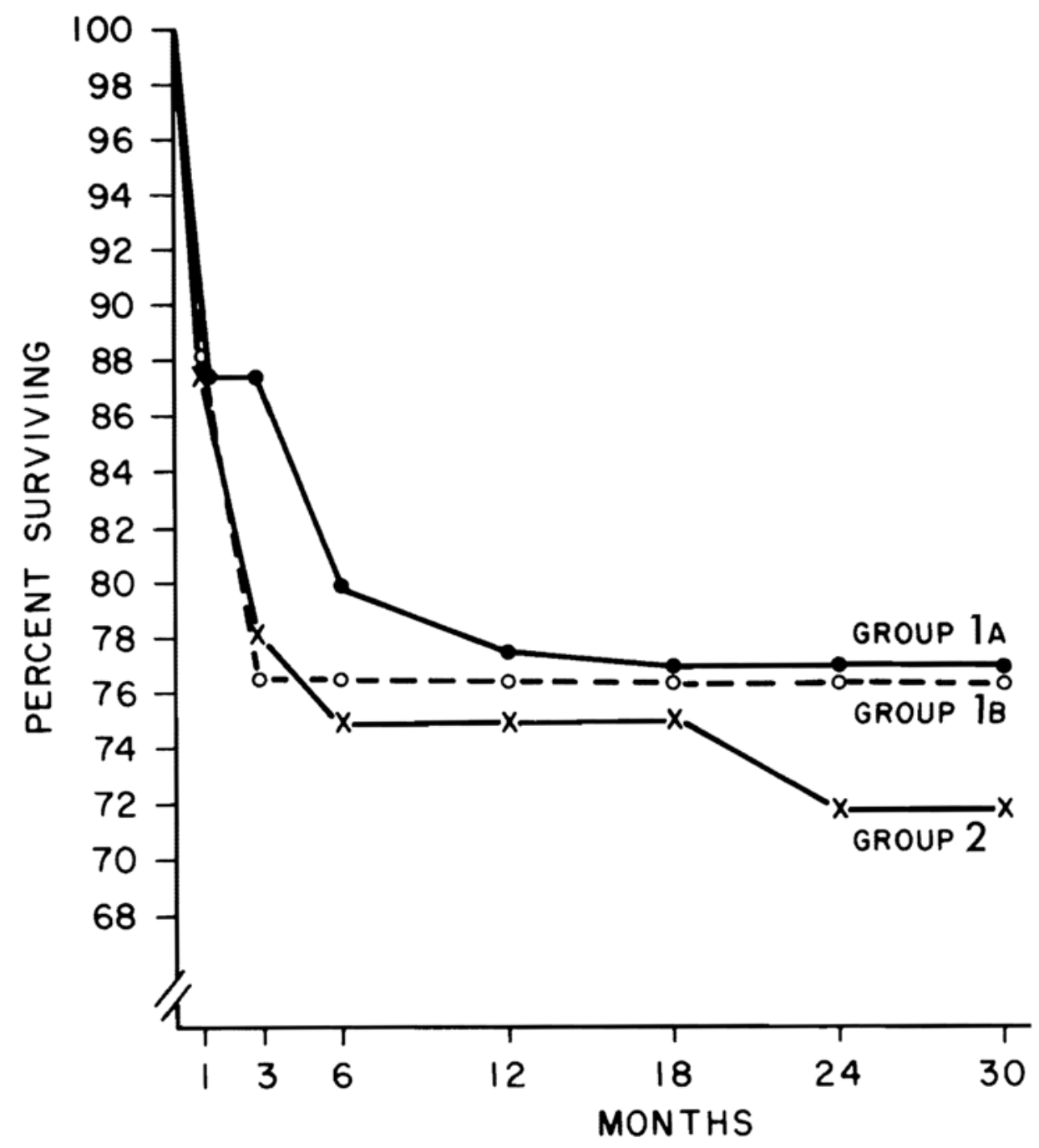

Figure 1.

Actuarial survival curves for the three groups of patients studied. Group 1A, those who had a prior Kasai procedure; group 1B, those who had prior abdominal surgery not including a Kasai procedure; and group 2, those without any prior surgery. No differences in survival are noted between the three groups for a period of 30 mo since the performance of the OLTx surgery. 
Table 1

Liver Disease for Which Orthotopic Liver Transplantation Was Applied in the Patients Studied

\begin{tabular}{lrlr}
\hline \multicolumn{1}{c}{ Group 1 } & \multicolumn{2}{c}{ Group 2 } \\
\hline Biliary atresia & 42 & $\alpha_{1}$-Antitrypsin deficiency & 12 \\
Chronic cholestasis other than biliary atresia & 7 & Chronic active hepatitis & 5 \\
& & Chronic cholestasis & 5 \\
Chronic active hepatitis & 4 & Cryptogenic cirrhosis & 3 \\
$\alpha_{1}$-Antitrypsin deficiency & 2 & Wilson's Disease & 3 \\
Cryptogenic cirrhosis & 1 & Hepatoma & 3 \\
Glycogen storage disease & 1 & Tyrosinemia & 1 \\
& & Fulminant hepatitis & 1 \\
\hline
\end{tabular}


Table 2

Preoperative Clinical and Laboratory Data in the Pediatric Patients Studied ${ }^{a}$

\begin{tabular}{lccc}
\hline & Group 1 (n= 57) & Group 2 (n= 32) & p \\
\hline Age $(\mathrm{mo})$ & $53 \pm 4$ & $88 \pm 11$ & $<0.001$ \\
Sex $(\mathrm{M} / \mathrm{F})$ & $21 / 36$ & $14 / 18$ & \\
Ascites $b$ & $24(42 \%)$ & $15(47 \%)$ & \\
Gastrointestinal hemorrhage $^{b}$ & $26(46 \%)$ & $1: 3(41 \%)$ & \\
Hepatic encephalopathy $b$ & $6(11 \%)$ & $8(25 \%)$ & \\
Serum bilirubin $(\mathrm{mg} / \mathrm{dl})$ & $18.7 \pm 1.6$ & $18.2 \pm 4.6$ & \\
Prothrombin time $(s)$ & $14.3 \pm 0.2$ & $16.3 \pm 0.7$ & $<0.01$ \\
Serum albumin $(\mathrm{g} / \mathrm{dl})$ & $3.5 \pm 0.2$ & $3.2 \pm 0.1$ & \\
Platelets $\left(\mathrm{No} . / \mathrm{mm}^{3}\right)$ & $190,725 \pm 200$ & $128,840 \pm 300$ & $<0.02$ \\
\hline
\end{tabular}

${ }^{a}$ Values are mean \pm SEM.

${ }^{b}$ Complication occurring before liver transplantation. 
Table 3

Incidence of Infections in Pediatric Patients Undergoing Orthotopic Liver Transplantation

\begin{tabular}{lccc}
\hline & Group 1 (n= 54) & Group 2 (n= 29) & p \\
\hline Patients with infections & $27(50 \%)$ & $8(28 \%)$ & $<0.05$ \\
Patients with abdominal infections & $19(35 \%)$ & $3(10 \%)$ & $<0.05$ \\
Number of abdominal infections & 23 & 3 & $<0.05$ \\
Wound infection & 9 & 1 & \\
Peritonitis & 4 & 1 & \\
Intraabdominal abscess & 4 & 1 & \\
Hepatic abscess & 3 & 0 & \\
Cholangitis & 3 & 0 & \\
Patients with nonabdominal infections & $13(24 \%)$ & $6(21 \%)$ & \\
Number of nonabdominal infections & 16 & 8 & \\
Bacteremia & 4 & 3 & \\
Candidemia & 3 & 1 & \\
Urinary tract infection & 3 & 1 & \\
Pneumonia & 2 & 0 & \\
Otitis & 2 & 0 & \\
Cytomegalovirus infection & 2 & 0 & \\
Herpes zoster & 0 & 2 & \\
Herpes simplex & 0 & & \\
& & & \\
\end{tabular}


Table 4

Pretransplanlation Clinical and Laboratory Data and Posttransplantation Costs and Course of the Pediatric Patients With a Previous Portoenterostomy (Group 1A) as Compared With Those With Other Previous Types of Abdominal Surgery (Group 1B) ${ }^{a}$

\begin{tabular}{|c|c|c|c|}
\hline & Group 1A $(n=40)$ & Group 1B (n = 17) & $\mathbf{p}$ \\
\hline Age (mo) & $43 \pm 3$ & $75 \pm 12$ & $<0.01$ \\
\hline $\operatorname{Sex}(M / F)$ & $15 / 25$ & $6 / 11$ & \\
\hline \multicolumn{4}{|l|}{ From first complaint to } \\
\hline \multicolumn{4}{|l|}{ OLTx } \\
\hline Ascites & $16(40 \%)$ & $8(47 \%)$ & \\
\hline Gastrointestinal hemorrhage & $16(40 \%)$ & $10(59 \%)$ & \\
\hline Hepatic encephalopathy & $5(12 \%)$ & $1(6 \%)$ & \\
\hline \multicolumn{4}{|l|}{ Immediately pre-OLTx } \\
\hline Serum bilirubin $(m g / d l)$ & $18.6 \pm 1.6$ & $18.8 \pm 4.6$ & \\
\hline Prothrombin time $(s)$ & $14.1 \pm 0.3$ & $14.8 \pm 0.4$ & \\
\hline Serum albumin $(g / d l)$ & $3.5 \pm 0.1$ & $3.6 \pm 0.3$ & \\
\hline Platelets $\left(\times 10^{3} / \mathrm{mm}^{3}\right)$ & $187 \pm 19$ & $198 \pm 35$ & \\
\hline \multicolumn{4}{|l|}{ During OLTx } \\
\hline Duration of anesthesia $(\mathrm{min})$ & $712 \pm 30$ & $665 \pm 25$ & \\
\hline Red blood cells transfused $(\mathrm{ml} / \mathrm{kg})$ & $247 \pm 35$ & $208 \pm 35$ & \\
\hline Fresh frozen plasma transfused $(\mathrm{ml} / \mathrm{kg})$ & $201 \pm 24$ & $181 \pm 35$ & \\
\hline Platelets transfused $(\mathrm{ml} / \mathrm{kg})$ & $15 \pm 3$ & $8 \pm 3$ & \\
\hline \multicolumn{4}{|l|}{ Post-OLTx infections } \\
\hline Patients with infection & $18(45 \%)$ & $9(53 \%)$ & \\
\hline Patients with abdominal infections & $13(33 \%)$ & $6(35 \%)$ & \\
\hline Patients with nonabdominal infections & $10(25 \%)$ & $3(18 \%)$ & \\
\hline Deaths & $5(12 \%)$ & $4(24 \%)$ & \\
\hline \multicolumn{4}{|l|}{ For survivors } \\
\hline Days in intensive care unit (post-OLTx) & $10 \pm 3$ & $15 \pm 8$ & \\
\hline Days in hospital (post-OLTx) & $52 \pm 4$ & $59 \pm 12$ & \\
\hline
\end{tabular}

OLTx, orthotopic liver transplantation.

${ }^{a}$ Values are mean \pm SEM. 